\title{
Spectral Fuzzy Classification System for Target Recognition
}

\author{
Ana Del Amo \\ Smiths Aerospace \\ Electronics Systems - Grand Rapids \\ Michigan, USA \\ ana.del.amo@smiths-aerospace.com
}

\author{
Daniel Gómez and Javier Montero \\ Dept. Statistics and O.R. \\ Complutense University \\ Madrid, Spain \\ monty@mat.ucm.es
}

\begin{abstract}
The goal of this paper is to present an algorithm for terrain matching, leveraging an existing fuzzy clustering algorithm, and modifying it to its supervised version, in order to apply the algorithm to georegistration and, later on pattern recognition.

Georegistration is the process of adjusting one drawing or image to the geographic location of a "known good" reference drawing, image, surface or map. The georegistration problem can be treated as a pattern recognition problem; and it can be applied to the target detection problem. The terrain matching algorithm will be based on fuzzy set theory as a very accurate method to represent the imprecision of the real world, and presented as a multicriteria decision making problem. The energy emitted and reflected by the Earth's surface has to be recorded by relatively complex remote sensing devices that have spatial, spectral and geometrical resolution constraints. Errors usually slip into the data acquisition process. Therefore, it is necessary to preprocess the remotely sensed data, prior to analyzing it (image restoration, involving the correction of distortion, degradation and noise introduced during the rendering process). In this paper we shall assume that all these problems have been solved, focusing our study on the image classification of a corrected image being close enough, both geometrically and radiometrically, to the radiant energy characteristics of the target scene. In particular, at a first stage we consider each pixel individually; and a class will be assigned to each pixel, taking into account several values measured in separate spectral bands. Then we shall describe an automatic detection system based on a previous algorithm developed in A. Del Amo et al. [3, 5], introducing now the fuzzy partition model proposed by A. Del Amo et al. $[2,4]$. A first phase will lead to a spectral definition of patterns; and a second phase will lead to classification and recognition. Similarity measures will then allow us to evaluate the degree to which a pixel can be associated to each pattern, or determine if a pattern is similar enough to
\end{abstract}

a subimage of the main image, to establish that a target we are looking for can be found on that image.

\section{Introduction}

In a recognition system, three pillars have to be taken into account: hardware, software and a collection of concepts, methods, and techniques based on soft computing that will allow automation of reasoning. Soft computing is a set of methodologies whose role model is the capability of the human mind to exploit the tolerance for imprecision, uncertainty and partial truth to achieve tractability, robustness and minimality of cost or effort.

Remotely sensed image classification is a extremely complex problem. Therefore, a usual approach is to divide it into more accessible mathematical problems. Some assumptions have to be made, as we are only going to work in a part of the recognition system. Let's assume that the images we will be working with have already been corrected. The Earth surface is amazingly complex; and it is not easy to record. The Earth surface has to be recorded by relatively complex remote sensing devices that have constraints such as spatial, spectral, geometric resolution. Errors usually slip into the data acquisition process. Therefore, it is usually necessary to preprocess the remotely sensed data, prior to analyzing it. This preprocessing part of the problem is usually called image restoration. Image restoration involves the correction of distortion, degradation, and noise introduced during the rendering process. Image restoration produces a corrected image that is as close as possible, both geometrically and radiometrically, to the radiant energy characteristics of the original scene. This can be considered as a whole different problem, which we are not going to consider here (a nice review can be found in [8]). In this paper we shall assume that the image has been already restored.

The simplest form of digital image classification is to consider each pixel individually, assigning it to a class based upon several values measured in separate spectral bands. 
This type of classifier is usually referred to as a spectral or point classifier. Although point classifiers offer benefits of simplicity and economy, they do not give the analyst the opportunity to exploit the information contained in relationships between each pixel and its neighbors.

By definition, a remotely sensed image illustrates a portion of the earth's surface as recorded by reflection of solar energy to an instrument at high altitude. The following questions illustrate the information necessary to interpret these images:

- What forms of radiation have been used to record this pattern?

- How was this radiation gathered and recorded as an image?

- What is the scale? (How large is the ground area represented in the image?)

- What ground patterns are represented by variations in brightness? (Are they related to others not visible?)

- When was the image acquired? (What time of day? What season?)

The field of Automatic Target Recognition (ATR) has been widely studied for at least two decades, showing a lack of appropriate mechanism to incorporate expert knowledge. In this sense, Fuzzy Logic provides a mechanism for incorporating expert knowledge into recognition systems. One difficulty with object recognition in images is the inability to define the objects in question. Definition is a prerequisite to recognition. Definability is concerned with whether and how a concept $X$ may be defined in a way that lends itself to mathematical analysis and computation.

A pattern refers to the arrangement of individual objects into distinctive, recurring forms that permit recognition on aerial imagery. Pattern on an image usually follows a functional relationship among the individual features that compose the pattern.

The definition of the patterns has to be done in the same mathematical model that the classification will be performed in. For this reason the system will have two phases: a definition phase, in which the patterns are spectrally defined, and a second phase which will be the classification and recognition.

\section{Georegistration}

Georegistration is the process of adjusting one drawing or image to the geographic location of a "known good" reference drawing, image, surface or map. For brevity, this topic and other georegistration topics use images as examples. However, the same procedures apply when georegistering drawings or surfaces.
The drawing, image, surface or map, being used as a reference is called the reference component. The drawing, image or surface being adjusted is called the target component.

Georegistration involves precise transformation of the image from the sensor based projection to an earth surface based projection. This process includes calculating a satellite mode, matching ground and image based control points and transformation and resampling the data to a map projection coordinate system. The most important factor to consider in geometric registration is the positional accuracy of each pixel as it is moved from the sensor based projection to the surface base projection.

Lets see some assumptions we will make for the reference component and for the target component.

\subsection{Assumptions}

Our ultimate objective is to build and demonstrate implementation of georegistration, using a high-speed Field Programmable Gate Array (FPGA) processing technique. Certain assumptions will be made and those include:

- Geo-referenced images with identified areas of interest for "correlation" will be already stored in memory.

- All images have been taken with the same resolution and have been stored in memory landscape.

- The set of images acquired for matching will be taken with the same resolution than the ones already in memory.

\section{Object classification}

Let

$$
X=\left\{X_{i j} / i=1, \cdots, r j=1, \cdots, s\right\}
$$

be the image set to classify, and each element $X_{i j}$ of the image set is considered to be an earth surface unit or pixel. Let

$$
\left(x_{i j}^{1}, x_{i j}^{2}, \cdots, x_{i j}^{n}\right) \in \mathbb{R}^{n}
$$

be the vectorial representation of a collection of features for element $X_{i j}$, in which $x_{i j}^{f}$ is the value of feature $f$ for element $X_{i j}$. These values could be a subproduct of a series of direct observations.

Most of the classification algorithms don't have an a priori number of classes. The number of classes it is usually set up after a set of comparisons between different classifications with a different number of classes set up a priory. In the case of a fuzzy classification the number of classes is considerably lower than in crisp classification due to the flexibility that a fuzzy class provides. We are working with an unknown number of classes $k$. For each one of 
the classes, in which the classification will be performed, a range of valid values has to be defined. The way it was done in the original algorithm follows (see [3]):

For each class $k$ and each feature $f$ let $\underline{\alpha}_{f k}$ and $\bar{\alpha}_{f k}$ be the lower and upper extremes of the interval $I_{f k}$, inside which the membership function has a value of 1 . Let $\underline{\omega}_{f k}$ and $\bar{\omega}_{f k}$ be the lower and upper extremes of the interval outside of which the membership function has a value of 0 . This means that element $X_{i j}$ crisply does not satisfy the $f$ property for class $C_{k}$ whenever $x_{i j}^{f}<\underline{\omega}_{f k}$ or $x_{i j}^{f}>\bar{\omega}_{f k}$; it satisfies the $f$ properties of class $C_{k}$ in a crisp way whenever $\underline{\alpha}_{f k} \leq x_{i j}^{f} \leq \bar{\alpha}_{f k}$; and it satisfies properties of class $k$ in a fuzzy way whenever $\underline{\omega}_{f k} \leq x_{i j}^{f}<\underline{\alpha}_{f k}$ or $\bar{\alpha}_{f k}<x_{i j}^{f} \leq \bar{\omega}_{f k}\left(\underline{\omega}_{f k}<\underline{\alpha}_{f k}<\bar{\alpha}_{f k}<\bar{\omega}_{f k}\right.$ should be verified).

Therefore, each object $X_{i j}$ has an associated vector

$$
M_{k}\left(X_{i j}\right)=\left(m_{1 k}\left(x_{i j}^{1}\right), m_{2 k}\left(x_{i j}^{2}\right), \cdots, m_{n k}\left(x_{i j}^{n}\right)\right)
$$

for each class $C_{k}$, which shows the different degrees of verification each property has with respect to each class.

Let $P=\left\{P_{1}, P_{2}, \cdots, P_{p}\right\}$ be the family of patterns. Each pattern $P_{u}$ will be a subset of objects characterized through the different observed features,

$$
P_{u}=\left\{\left(P_{u}\right)_{i, j} / i=1, \cdots, r_{k} j=1, \cdots, s_{k}\right\}
$$

verifying $1 \leq r_{p} \leq r$ and $1 \leq s_{p} \leq s$. Then,

$$
\left(\left(p_{u}\right)_{i j}^{1},\left(p_{u}\right)_{i j}^{2}, \cdots,\left(p_{u}\right)_{i j}^{n}\right) \in \mathbb{R}^{n}
$$

is the vectorial representation of each one of the pixels in the pattern, where $\left(p_{u}\right)_{i j}^{f}$ is the value of feature $f$ for pixel $\left(p_{u}\right)_{i j}$ in the subset $P_{u}$.

Each pixel in the pattern $P_{u}$ has been already classified or spectrally defined using the unsupervised classification system presented in [3] and [5]. So each one of the pixels in the pattern image will have a representation as follows:

$$
\mu_{i j}^{u}\left(C_{k}\right)=\mu_{i j}^{u^{N D}}\left(C_{k}\right) \cdot b_{i j}\left(C_{k}\right)
$$

as the membership degree to class $C_{s}$.

Where:

- $C=\left\{C_{1}, C_{2}, \cdots, C_{b}\right\}$ is the family of classes. Each class $C_{k}$ will become characterized through the different observed features.

- $\mu_{i j}^{u^{N D}}\left(C_{k}\right)$ the fuzzy set of non-dominated classes, it represents the membership degree of pixel $X_{i j}$ to class $C_{k}$.

- $b_{i j}\left(C_{k}\right)$ basic pixel information ${ }^{1}$.

$$
\begin{aligned}
& \text { 'each object }\left(P_{u}\right)_{i j} \text { has an associated vector } \\
& M_{t}\left(\left(P_{u}\right)_{i j}\right)=\left(m_{1 t}\left(\left(p_{u}\right)_{i j}^{1}\right), m_{2 t}\left(\left(p_{u}\right)_{i j}^{2}\right), \cdots, m_{n t}\left(\left(p_{u}\right)_{i j}^{n}\right)\right)
\end{aligned}
$$

for each class $C_{t}$, which shows the different degrees of verification each property has with respect to each class.
Pixel $X_{i j}$ will verify the properties of class $C_{k}$ with an intensity of

$$
b_{i j}\left(C_{k}\right)=\min _{f}\left\{m_{f s}\left(x_{i j}^{f}\right)\right\}
$$

Where $m_{f s}\left(x_{i j}^{f}\right)$ is the membership function for each class (as defined above) $C_{u}$ with respect to each $f$ property.

\section{Fuzzy Pattern Recognition System}

The fuzzy pattem recognition system that we will present here operates in a two pass mode (i.e., it passes through the registered multispectral dataset twice). In the first pass, the program reads through the dataset and sequentially build a fuzzy classification of each pixel in the image. On the other hand, a set of objects has been individually classified by the same method. In the second pass, a comparison between a pattern and the image we are classifying will be performed.

The definition of a pattem after its classification using the fuzzy classification system will be as follows:

$$
P_{k}^{d e f}=\left\{\mu_{i j}^{k}\left(C_{u}\right) / C_{u} \in\left\{C_{1}, \cdots, C_{b}\right\}\right\}
$$

A fundamental issue to take into account when a spectral classification has been performed is that a difference exists in the spectral response of a pixel on two dates if the biophysical materials within the Instantaneous Field Of View (IFOV) have changed between dates. Ideally, the spectral resolution of the remote sensor system is sufficient to record reflected radiant flux in spectral regions that best capture the most descriptive spectral attributes of the object.

Once the sets of patterns and the whole set of pixels in the image have been spectrally classified or defined, the recognition phase starts. Each one of the patterns has to be compared with the image that is being used to locate the target. Once, the size of the pattern $k$ has been determined, (let's say $r_{k} \times s_{k}$ for pattern $k$ ) we will have to go through the image comparing chips of size $r_{k} \times s_{k}$ all over the image. This can be done just comparing the first line of pixels $r_{k}$ in the pattern with the image sliding the pattern over the image starting on the left top corner. Another possibility could be to create a filter and use the filter in the whole set of images. The filter will be use to determined possible sets of pixels in a target image and in the acquired image that can be considered as similar for a particular similarity relation.

A similarity measure has been used in the first place to decide whether or not two objects are members of the same cluster. Now a similarity measure has to be defined to determine if a pattern image or part of it is similar enough to a subset of the acquired image to be able to either determined that a target has been detected or to be able to match the geographic location of a set of pixels in the acquired image. 
It is very important to consider the neighbors of each pixel in order to perform a classification. Some of the relations between pixels can only be found through the study of the neighbors and very many times some of the errors due to some acquisition difficulties can be solved or not be considered only if the neighbors have been considered in the analysis. As has been established above a classification of the pixels in the pattern images has been already performed. Therefore, we have a classification pixel by pixel to each one of the classes in the class set (each matrix $\Gamma_{C_{k}}$ represent each one of the individual pixel as a vector representing the degrees of membership of the pixel to each one of the classes in the classification). Let $\Gamma$ be the image already classified using Del Amo et al. algorithm as follows 2). The set:

$$
\Gamma=\left\{\Gamma_{C_{k}} / k=\{1, \cdots, b\}\right\}
$$

where $\Gamma_{C_{k}}$ is defined as follows:

$$
\Gamma_{C_{k}}=\left\{\rho \mu_{i j}\left(C_{k}\right) \quad i=1, \cdots, r ; j=1, \cdots, s\right\}
$$

$\Gamma$ as have been defined above, represent the classification of the original image by the Del Amo et al. algorithm. The second part of the algorithm will consider the neighbors. It won't be a pixel by pixel classification; we need to consider not only the particular characteristics of each pixel but also their location in the image.

From this, we will compute a transformation matrix. The transformation matrix will be the result of the application of a filter which is intended to exaggerate differences. The resultant matrix will be call $\Xi$-transformation.

Once the transformation matrix has been calculated, we will proceed to define what we are going to call the geonumber:

Definition 4.1 A Individual Geo-number of size q for class $k$ and pixel $(i, j)$ or a geo $-q$ number will be the determinant of the $q \times q$ sub-matrix obtained after the $\Xi$-transformation of size $q$ has been performed.

$$
\text { Ind }-G e o_{-q}(i, j, k)=\left.||\left[\rho \mu_{i j}\left(C_{k}\right)\right]\right|_{q} \mid
$$

Definition 4.2 A Group-Geo-number of size $q$ for class $k$ around pixel $(i, j)$ be the minimum of the Individual Geonumbers of the $(q \times q-1)$-neighbors.

$$
\text { Group }-G e o_{-q}(i, j, k)=\left\{\left.\min _{(q \times q-1) \text { neigh }}||\left[\rho \mu_{i j}\left(C_{k}\right)\right]\right|_{q} \mid\right\}
$$

Definition 4.3 A Geo-number of size $q$ for $(i, j)$ would be the maximum of the Group-Geo-numbers of the $(q \times q-1)$ neighbors.

$$
\mathrm{Geo}_{-q}(i, j)=\left\{\max _{(q \times q-1) \text { neigh }} \text { Group }-\mathrm{Geo}_{q}\right\}
$$

${ }^{2}$ When we consider $\Gamma$ for a pattern image we will use the notation $\Gamma_{p}$; and the elements of the matrix will be noted $\rho_{p} \mu_{i j}\left(C_{k}\right)$
In the first place we will define when two sets of pixels from two different images will be comparable or not. Once, a comparability threshold has been determined, a similarity relation can be defined.

Also, a comparability degree can be defined that will represent the degree of accuracy that we are looking for. A sensibility analysis can be performed in order to obtain a reasonable level of accuracy not compromising the number of false detections included.

\section{Detecting Similarities}

The target recognition system that will be developed here will have the option of real time selection of possible target areas. The user will be able to select a possible candidate area to be a target. The selected area will be compared with the images stored in memory for possible matching. The matching will be performed as follows: The selected area in the read image will be classified using the unsupervised fuzzy classification algorithm. After the classification has been performed the $\Xi$-transformation and also the $\mathrm{Geo}_{q^{-}}$ numbers starting from the upper left corner of the selected area will be calculated. The algorithm will scan the images in the search for similar $\mathrm{Geo}_{q}$-numbers.

Given any two image sets $I_{1}$ and $I_{2}$, and $\Xi$ - transformations for $I_{1}$ and $I_{2}$ respectively, we will determine whether a $q$-neighborhood of a pixel in a selected area is similar to the same size neighborhood in the stored image by looking at their respective $\mathrm{Geo}_{q}$-numbers. The degree of similarity between $\mathrm{Geo}_{q}$-numbers will give us a degree of accuracy in our assessment.

\section{An improved algorithm}

The original unsupervised classification algorithm proposed by the authors was applied to a remotely sensed image obtained from Sevilla surroundings, south Spain (see [3] for a description of the unsupervised algorithm and [5] for an application to a real digital image). Results were considered extremely good, as the representations included a natural description of vegetation cover (forest, scrubland, swampland, etc.) The advantage of a fuzzy approach against a classical crisp approach was obvious: that particular picture was quite well explained assuming the existence of a few fuzzy classes, allowing of course natural mixtures between those in between classes, showing in addition a natural structure of our classification system. The results not only showed a list of classifications, but also identified some relationships between the classification of different areas. Moreover, results obtained by means of a few fuzzy classes needed quite a bigger number of crisp classes in order to get equivalent results; and a naive look at the considered image 
showed the existence of three main fuzzy classes, with no sharp boundaries among them. When talking about Earth vegetation cover, fuzzy classification may be much more natural and accurate than a corresponding crisp classification. The key issue is the conceptual accuracy given by fuzzy classes. If reality shows natural fuzzy classes, with no crisp borders' but gradation between classes, we should expect that fuzzy approaches will be more accurate.

In this paper we incorporate into the previous algorithm some tools designed in [7] relative to aggregation of neighbor pixels, based upon the recursive approach [6], now translated to the real surface (instead of the real line). A first application to a satellite image obtained from the Sierra of Madrid showed the need for standard statistical multivariate techniques; but at the same time we were able to obtain some knowledge about how to improve our basic classification system [4], introducing degrees of covering, relevancy and overlapping, in the sense of [2]. In this way we can properly learn.

Now, the above considerations allow additional implementations that open the possibility of applying the improved algorithm to target detection. Such a modification is tried in both digital images of Sevilla and Madrid.

\section{Final comments}

The goal of this research is to build up a model for classification whenever a comparative analysis is an essential part of the available information. In particular, we pursue an automatic detection system based on a previous algorithm developed by Del Amo et al., which was the core of a classification system based on fuzzy set theory (see [12]). In this way we can gain the advantage of non probabilistic imprecision (entities with no sharp borders), in the automated process of assigning image areas to pre-defined surface types. Such an algorithm was presented by the authors as a multicriteria decision making problem, by means of an outranking methodology [10], as considered also in [9].

The original algorithm was a unsupervised classification algorithm based on modified classic outranking methods in order to fit the objective of the model. This paper offers key modifications on the original algorithm in order to allow its supervised version plus its application to georegistration and target detection.

Acknowledgements: This research has been partially supported by Smiths Aerospace as an $I R \& D$ project and by the Government of Spain, research grant BFM2002-0281.

\section{References}

[1] J.C. Bezdek, and S. K. Pal: Fuzzy Models for Pattern Recognition. IEEE Press, New York, 1992.
[2] A. Del Amo, D. Gómez, J. Montero and G. Biging: "Relevance and redundancy in fuzzy classification systems". Mathware and Soft Computing 8:203-216, 2001.

[3] A. Del Amo, J. Montero and G. Biging: Classifying pixels by means of fuzzy relations. International Journal of General Systems 29:605-621, 1999.

[4] A. Del Amo, J. Montero, G. Biging and V. Cutello: "Fuzzy classification systems". European Journal of Operational Research, to appear.

[5] A. Del Amo, J, Montero, A. Fernandez, M. Lopez, J. Tordesillas and G. Biging: "Spectral fuzzy classification: an application". IEEE Trans on Systems, Man and Cybernetics (C) 32:42-48, 2002.

[6] A. Del Amo, J. Montero, and E. Molina: "Representation of consistent recursive rules". European Journal of Operational Research 130:29-53, 2001.

[7] D. Gómez: "Algunas aportaciones sobre relaciones de preferencia". Ph.D. Thesis, Complutense University of Madrid, Spain (in Spanish).

[8] J. R. Jensen: Introductory Digital Image Processing. A Remote Sensing Perspective. Prentice Hall, New York, 1986.

[9] A.D. Pearman, J. Montero and J. Tejada: "Fuzzy multicriteria decision support for budget allocation in the transport sector". TOP 3:47-68, 1995.

[10] P.P. Perny and B. Roy: "The use of fuzzy outranking relations in preference modelling". Fuzzy Sets and Systems 49:3-53, 1992.

[11] J. Siskos, J. Lochard and J. Lombard: "A multicriteria decision making methodology under fuzziness: application to the evaluation of radiological protection in nuclear power plants". In: H.J.Zimmermann, L.A. Zadeh and B.R. Gaines (eds.), Fuzzy Sets and Decision Analysis. North Holland, Amsterdam, 1984.

[12] L. A. Zadeh: "Fuzzy Sets". Information and Control 8:338-353, 1965. 